\title{
THE LONG OF IT: ODDS THAT INVESTOR SENTIMENT SPURIOUSLY PREDICTS
} ANOMALY RETURNS

\author{
Robert F. Stambaugh \\ Jianfeng Yu \\ Yu Yuan
}

Working Paper 18231

http://www.nber.org/papers/w18231

\author{
NATIONAL BUREAU OF ECONOMIC RESEARCH \\ 1050 Massachusetts Avenue \\ Cambridge, MA 02138 \\ July 2012
}

The views expressed herein are those of the authors and do not necessarily reflect the views of the National Bureau of Economic Research.

NBER working papers are circulated for discussion and comment purposes. They have not been peerreviewed or been subject to the review by the NBER Board of Directors that accompanies official NBER publications.

(C) 2012 by Robert F. Stambaugh, Jianfeng Yu, and Yu Yuan. All rights reserved. Short sections of text, not to exceed two paragraphs, may be quoted without explicit permission provided that full credit, including (C) notice, is given to the source. 
The Long of It: Odds that Investor Sentiment Spuriously Predicts Anomaly Returns

Robert F. Stambaugh, Jianfeng Yu, and Yu Yuan

NBER Working Paper No. 18231

July 2012

JEL No. C18,G12,G14

\begin{abstract}
Extremely long odds accompany the chance that spurious-regression bias accounts for investor sentiment's observed role in stock-return anomalies. We replace investor sentiment with a simulated persistent series in regressions reported by Stambaugh, Yu and Yuan (2012), who find higher long-short anomaly profits following high sentiment, due entirely to the short leg. Among 200 million simulated regressors, we find none that support those conclusions as strongly as investor sentiment. The key is consistency across anomalies. Obtaining just the predicted signs for the regression coefficients across the 11 anomalies examined in the above study occurs only once for every 43 simulated regressors.
\end{abstract}

Robert F. Stambaugh

Finance Department

The Wharton School

University of Pennsylvania

Philadelphia, PA 19104-6367

and NBER

stambaugh@wharton.upenn.edu

Jianfeng Yu

University of Minnesota

321 19th Avenue South, Suite 3-122

Minneapolis MN 55455

jianfeng@umn.edu
Yu Yuan

The University of Pennsylvania

3620 Locust Walk Suite 2300

Philadelphia, PA 19104

yuanyu@wharton.upenn.edu 


\section{Introduction}

Caution is warranted when inferring that a highly autocorrelated variable has the ability to predict asset returns. One reason is the possibility of a "spurious" regressor: If the unobserved expected return on an asset is time-varying and persistent, another persistent variable having no true relation with return can appear to predict return in a finite sample. Ferson, Sarkissian, and Simin (2003) demonstrate how the potential for such regressors complicates the task of assessing return predictors, and they explain how the underlying mechanism relates to the spurious regression problem analyzed by Yule (1926) and Granger and Newbold (1974). Ferson, Sarkissian, and Simin (2003) also explain how data mining interacts with the problem of spurious regressors. When the potential for spurious regressors exists (i.e., a persistent time-varying expected return), then the more series researchers search through, the more likely they are to find a series that appears to predict returns but does so only spuriously.

The greater is the prior motivation for entertaining a series as a return predictor, the less is the concern that its apparent predictive ability is spurious. For example, such a concern is likely to be lower when the predictor is a risk measure or a valuation ratio, as opposed to, say, sunspots. ${ }^{1}$ One quantity with strong prior motivation as a return predictor is market-wide investor sentiment. At least as early as Keynes (1936), numerous authors have considered the possibility that a significant presence of sentiment-driven investors can cause prices to depart from fundamental values, thereby creating a component of future returns that corrects such mispricing. Baker and Wurgler (2006) and Stambaugh, Yu and Yuan (2012), among others, find that investor sentiment and/or consumer confidence exhibits an ability to predict returns on various classes of stocks and investment strategies. ${ }^{2}$ These studies also refine the prior motivation of investor sentiment as a predictor. For example, Baker and Wurgler (2006) argue that sentiment should play a stronger role among stocks that are more difficult to value. In support of that hypothesis, they find sentiment exhibits greater ability to predict returns on small stocks, young stocks, high volatility stocks, unprofitable stocks, nondividend-paying stocks, extreme growth stocks, and distressed stocks. Stambaugh, Yu, and

\footnotetext{
${ }^{1} \mathrm{~A}$ regressor with prior motivation also often violates the spurious-regressor setting in Ferson, Sarkissian, and Simin (2003), wherein the regressor bears no relation to return. Instead, the innovation in the regressor is often correlated with contemporaneous return, whether or not the regressor predicts future return. Such a correlation is especially likely for a regressor that is a valuation ratio, such as dividend yield. The finitesample bias that arises in such a setting is analyzed by Stambaugh (1999).

${ }^{2}$ Other studies that document the ability of sentiment measures to predict returns include Brown and Cliff (2004, 2005), Lemmon and Portniaguina (2006), Baker and Wurgler (2007), Livnat and Petrovic (2008), Yu (2009), Antoniou, Doukas, and Subrahmanyam (2010), Gao, Yu, and Yuan (2010), and Baker, Wurgler, and Yuan (2012).
} 
Yuan (2012) hypothesize that when market-wide sentiment is combined with Miller's (1977) argument about the effects of short-sale impediments, overpricing due to high sentiment is more likely than underpricing due to low sentiment. Their results support that argument, in that sentiment predicts profits on the short legs of a large set of anomaly-based long-short strategies, whereas sentiment exhibits no ability to predict long-leg profits.

Despite the prior motivations for the properties that investor sentiment exhibits empirically as a predictor, some might nevertheless be concerned that sentiment is simply a spurious predictor. Indeed, such a concern is the focus of Novy-Marx (2012). This study assesses the odds that investor sentiment's observed ability as a predictor can be achieved by a spurious regressor. We focus on the role of consistency across multiple return series and hypotheses. To understand the value of consistency, suppose the true expected returns across a number of portfolios possess some independent variation, but each expected return's true correlation with investor sentiment has the same sign. The greater the number of portfolios, the more difficult it becomes to find a spurious regressor that will exhibit finite-sample predictive ability consistently across portfolios comparable to that of investor sentiment. Our setting for exploring the role of consistency is that of Stambaugh, Yu, and Yuan (2012). That study examines 11 different anomalies and finds consistent results across those anomalies in support of three hypotheses: (i) a positive relation between current sentiment and future long-short return spreads, (ii) a negative relation between current sentiment and future short-leg returns, and (iii) no relation between current sentiment and future long-leg returns. We simply ask how likely it is that such hypotheses are supported as strongly by a randomly generated spurious regressor used in place of investor sentiment.

Out of 200 million simulated regressors, we find none that jointly support the three hypotheses in Stambaugh, Yu, and Yuan (2012) as strongly as investor sentiment. The odds are still quite long if one looks at just one of the three hypotheses. For example, comparably strong and consistent support for the first hypothesis - a positive relation between sentiment and the long-short return spread-occurs once in every 28,500 simulated regressors. For the second hypothesis - a negative relation between sentiment and short-leg returns - comparable support occurs once in every 105,000 regressors. If one sets aside any consideration of strength (t-statistics) and simply looks at the signs of regression coefficients dictated by the first two hypotheses, even then only one in every 43 simulated regressors achieves the consistency exhibited with investor sentiment.

The remainder of the paper proceeds as follows. Section 2 reviews the regression setting and original empirical results, describes the process for generating spurious regressors, and 
presents the simulation results. Section 3 concludes.

\section{Empirical setting and simulation results}

The empirical setting we analyze here focuses on the main set of regression results reported by Stambaugh, Yu, and Yuan (2012), hereafter SYY. That study estimates the regression,

$$
R_{i, t}=a+b S_{t-1}+c M K T_{t}+d S M B_{t}+e H M L_{t}+u_{t}
$$

where $R_{i, t}$ is the excess return in month $t$ on an anomaly strategy's long leg, short leg, or the difference, $S_{t-1}$ is the level of the investor-sentiment index of Baker and Wurgler (2006) at the end of month $t-1$, and $M K T_{t}, S M B_{t}$, and $H M L_{t}$ are the returns on month $t$ on the three stock-market factors defined by Fama and French (1993). SYY examine 11 anomalies documented previously in the literature:

1. Failure probability (Campbell,Hilscher, and Szilagyi, 2007)

2. Distress (Ohlson, 1980)

3. Net stock issues (Ritter, 1991, and Loughran and Ritter, 1995)

4. Composite equity issues (Daniel and Titman, 2006)

5. Total accruals (Sloan, 1996)

6. Net operating assets (Hirshleifer, Hou, Teoh, and Zhang, 2004)

7. Momentum (Jegadeesh and Titman, 1993)

8. Gross profitability (Novy-Marx, 2010)

9. Asset growth (Cooper, Gulen, and Schill, 2008)

10. Return on assets (Fama and French, 2006, Chen, Novy-Marx, and Zhang, 2010, Wang and $\mathrm{Yu}, 2010)$

11. Investment-to-assets (Titman, Wei, and Xie, 2004, and Xing, 2008)

As in SYY, the sample period is from August 1965 through January 2008 for all but anomaly (1), whose data begin in December 1974, and anomalies (2) and (10), whose data begin in January 1972. For each anomaly, SYY examine the long-short strategy using deciles 1 and 10 of a sort based on the anomaly variable, with the long leg being the decile with the highest average return. SYY also examine a combination strategy that takes equal positions across the long-short strategies constructed in any given month.

The coefficient of interest in equation (1) is b. SYY (cf. table 5) report results of estimating $b$ for each of the 11 anomalies, as well as the combination strategy, in three 
sets of regressions that relate to the three hypotheses explored in that study. For the first hypothesis, $R_{i, t}$ is the long-short return difference, and the estimate $\hat{b}$ has the predicted positive sign for all 11 anomalies. The t-statistic for $\hat{b}$, based on the heteroskedasticityconsistent standard error of White (1980), ranges from 0.22 to 3.38 across the individual anomalies and equals 2.98 for the combination strategy. For the second hypothesis, $R_{i, t}$ is the short-leg return, and $\hat{b}$ has the predicted negative sign for all 11 anomalies. The t-statistic ranges from -1.11 to -3.58 across the individual anomalies and equals -3.01 for the combination strategy. The third hypothesis, in which $R_{i, t}$ is the long-leg return, predicts $b$ should be roughly zero. In these regressions, the signs of $\hat{b}$ are mixed across the individual anomalies ( 7 positive, 4 negative), with t-statistics ranging from -2.07 to 1.44 , and the combination strategy has a t-statistic of 0.15 . When viewed collectively across the estimated 36 regressions (12 for each hypothesis), the SYY results appear to present fairly strong support for all three hypotheses explored.

In this study, we ask how likely it is that a spurious predictor would support the three SYY hypotheses as strongly as investor sentiment. We randomly generate a predictor series $x_{t}$, use it to replace $S_{t}$, and then re-estimate equation (1) for the same 36 regressions summarized above. That procedure is repeated 200 million times. Each predictor series $x_{t}$ is generated as a first-order autoregressive process with normal innovations and autocorrelation equal to 0.988 , which equals the sample autocorrelation of $S_{t}$ adjusted for the first-order bias correction in Marriott and Pope (1954) and Kendall (1954).

To judge whether $x_{t}$ supports a given hypothesis as strongly as $S_{t}$, we ask whether the t-statistics for $\hat{b}$, viewed jointly across anomalies, are as favorable to the hypothesis as those produced using $S_{t}$. To determine this condition in the case of the first hypothesis, for which $R_{i, t}$ is the long-short return difference, define $\bar{t}_{i}^{S}$ as the $i$-th highest t-statistic for $\hat{b}$ among the 11 anomalies when $S_{t}$ is used. Similarly define $\bar{t}_{i}^{x}$ as the $i$-th highest t-statistic for $\hat{b}$ among the 11 anomalies when $x_{t}$ is used. Let $t_{C}^{S}$ denote the t-statistic for the combination strategy when $S_{t}$ is used, and let $t_{C}^{x}$ denote the corresponding t-statistic when $x_{t}$ is used. Then $x_{t}$ supports the first hypothesis $(b>0)$ as strongly as $S_{t}$ if $\bar{t}_{i}^{x} \geq \bar{t}_{i}^{S}$ for $i=1, \ldots, 11$ and $t_{C}^{x} \geq t_{C}^{S}$.

Only once in every 28,500 generated $x_{t}$ series, on average, is the first hypothesis supported as strongly by $x_{t}$ as by $S_{t}$. This result is reported in the last row of the first column of Table 1. The other rows display the frequencies with which fewer of the above inequalities are satisfied. For example, the first row of the same column reports that at least one of the 11 values of $\bar{t}_{i}^{x}$ exceeds the corresponding value of $\bar{t}_{i}^{S}$ once in each 22 generated $x_{t}$ series. The 
sharp increase in values as one moves down the column illustrates the dramatic effect of requiring consistency across multiple anomalies. Just finding an $x_{t}$ for which more than half of the $\bar{t}_{i}^{x}$ values exceed the corresponding $\bar{t}_{i}^{S}$ values happens only once in every $833 x_{t}$ series. The next-to-last row reports that, for just the combination strategy, the t-statistic obtained with $x_{t}$ exceeds that obtained with $S_{t}$ once in every 67 series.

The odds for a spurious regressor become even longer when considering the second hypothesis, as we see from the second column of Table 1. That hypothesis is supported as strongly by $x_{t}$ as it is by $S_{t}$ only once in every 105,000 series. The inequality conditions here are essentially just the reverse of those earlier, since $R_{i, t}$ is now the short-leg return and the prediction is instead that $b<0$. Let $\underline{t}_{i}^{S}$ denote the $i$-th lowest t-statistic for $\hat{b}$ when $S_{t}$ is used, and let $\underline{t}_{i}^{x}$ denote the $i$-th lowest t-statistic when $x_{t}$ is used. Then $x_{t}$ supports the second hypothesis as strongly as $S_{t}$ if $\underline{t}_{i}^{x} \leq \underline{t}_{i}^{S}$ for $i=1, \ldots, 11$ and $t_{C}^{x} \leq t_{C}^{S}$. As with the first hypothesis, the effects of requiring consistency across the separate regressions are dramatic. Even for just the single regression with the combination strategy, however, obtaining a negative t-statistic greater in magnitude than that obtained with $S_{t}$ occurs only once in every 169 series.

The third hypothesis is that $b=0$. In order for that hypothesis to be supported at least as strongly by a randomly generated $x_{t}$ as it is by $S_{t}$, we require $x_{t-1}$ to be as consistently weak as $S_{t-1}$ in its ability to predict $R_{i, t}$, now defined as the long-leg return. For this case, let $|t|_{i}^{S}$ denote the $i$-th smallest t-statistic in absolute value when $S_{t}$ is used, and let $|t|_{i}^{x}$ denote the $i$-th smallest t-statistic in absolute value when $x_{t}$ is used. Then $x_{t}$ supports the third hypothesis as strongly as $S_{t}$ if $|t|_{i}^{x} \leq|t|_{i}^{S}$ for $i=1, \ldots, 11$ and $\left|t_{C}^{x}\right| \leq\left|t_{C}^{S}\right|$.

While the odds for a spurious regressor improve when considering just the third hypothesis, they are still rather long. Again we see the effect of consistency when requiring the absence of an apparent relation with the regressor. Only once in every 919 randomly generated $x_{t}$ series do we find one that is as consistently unsuccessful in predicting long-leg returns.

Of course, the story does not end with simply considering each of the three hypotheses in isolation. As SYY explain, these hypotheses arise as a set of joint implications, developed by combining the presence of market-wide swings in sentiment with the argument in Miller (1977) that short-sale impediments allow overpricing to be more prevalent than underpricing. The final two columns report the frequencies with which a spurious regressor $x_{t}$ supports more than one hypothesis as strongly as $S_{t}$, where comparable support of each individual hypothesis is judged as before. Only one spurious regressor out of 468,000 supports the first 
two hypotheses as strongly as investor sentiment. When we look for a spurious regressor that supports all three hypotheses as strongly as investor sentiment, we actually find none among 200 million simulated series. When confining the exercise to just the single regressions using the combination strategy, we still find that only one spurious regressor out of every 6,580 simultaneously supports each of the three hypotheses as strongly as investor sentiment.

Fairly unlikely is just the possibility that a spurious regressor would give $\hat{b}$ 's with the predicted signs consistently across all anomalies. Table 2 reports the frequencies with which a spurious regressor gives the predicted sign across anomalies for the long-short difference (first hypothesis) and the short-leg return (second hypothesis). For the first hypothesis, one in every 25 spurious regressors gives the predicted positive sign for all 11 anomalies. For the second hypothesis, the frequency of getting the predicted negative sign for all 11 anomalies is one in every 21. A spurious predictor that produces all 22 coefficients with the predicted signs, as does investor sentiment, occurs only once in every 43 randomly generated regressors.

Finally, our approach comparing the $i$-th strongest t-statistic using $x_{t}$ to the $i$-th strongest using $S_{t}$ is not the only way to demonstrate the importance of consistency across multiple anomalies. One might instead, for example, focus on just the single anomaly out of the 11 for which the t-statistic provides the weakest support for a hypothesis. For the first hypothesis, which predicts $b>0$ when $R_{i, t}$ is the long-short return difference, the lowest t-statistic produced by $S_{t}$ among the 11 anomalies is equal to 0.22 . Less than one $x_{t}$ series out of each 50 produces a minimimum t-statistic greater than 0.22 . For the second hypothesis, which predicts $b<0$ when $R_{i, t}$ is the short-leg return, the maximum t-statistic produced by $S_{t}$ is -1.11 (all other t-statistics are greater in negative magnitude). Less than one $x_{t}$ series out of 2500 produces a maximum t-statistic lower than that value. Thus, comparing just the weakest results across the individual anomalies also reveals that it is rather unlikely that a spurious regressor can replace investor sentiment.

\section{Conclusions}

It seems extremely unlikely that the observed role of investor sentiment in stock-return anomalies can be filled by a spurious regressor. Out of 200 million simulated regressors, we find none. These very long odds - seemingly no better than those attached to winning the Powerball Jackpot with a single play - reflect the consistency with which investor sentiment 
produces results across multiple anomalies for three hypotheses. ${ }^{3}$ Simultaneous support of those hypotheses is important, by itself, in that the odds of a spurious regressor supporting them as strongly as investor sentiment are only 1 in 6,580 even when all of the anomalies are combined into a single long-short strategy. It is the consistency across the individual anomalies, however, that raises the highest hurdle for a spurious regressor to clear in order to play the role of investor sentiment.

\footnotetext{
${ }^{3}$ Powerball is a multi-state lottery in which the odds of a single combination of numbers claiming a share of the top "Jackpot" prize are roughly 1 in 175 million.
} 


\section{Table 1 \\ Number of Randomly Generated Predictors Required to Obtain One Predictor That Produces Results as Strong as Investor Sentiment}

The table reports the reciprocal of the frequency with which a randomly generated predictor $x_{t}$ produces results as strong as investor sentiment $S_{t}$ when $x_{t}$ replaces $S_{t}$ in the regression,

$$
R_{i, t}=a+b S_{t-1}+c M K T_{t}+d S M B_{t}+e H M L_{t}+u_{t},
$$

where $R_{i, t}$ is the excess return in month $t$ on an anomaly's long leg, short leg, or the difference, $S_{t}$ is the level of the investor-sentiment index of Baker and Wurgler (2006), and $M K T_{t}, S M B_{t}$ and $H M L_{t}$ are the three stock-market factors defined in Fama and French (1993). The predictor $x_{t}$ is generated as a first-order autoregression with autocorrelation equal to 0.988 , the bias-corrected estimate of the autocorrelation of $S_{t}$.

Let $\bar{t}_{i}^{S}$ denote the $i$-th highest t-statistic for $\hat{b}$ (the estimate of $b$ ) among the 11 anomalies when $S_{t}$ is used, and let $\bar{t}_{i}^{x}$ denote the $i$-th highest t-statistic when $x_{t}$ is used. Let $\underline{t}_{i}^{S}$ denote the $i$-th lowest t-statistic for $\hat{b}$ when $S_{t}$ is used, and let $\underline{t}_{i}^{x}$ denote the $i$-th lowest t-statistic when $x_{t}$ is used. Let $|t|_{i}^{S}$ denote the $i$-th smallest t-statistic in absolute value when $S_{t}$ is used, and let $|t|_{i}^{x}$ denote the $i$-th smallest t-statistic in absolute value when $x_{t}$ is used. The row for $j$ anomalies reflects the frequency with which the following conditions are satisfied:

$$
\begin{aligned}
& \bar{t}_{i}^{x} \geq \bar{t}_{i}^{S} \text { occurred at least } j \text { times among } i=1, \ldots, 11 \text {, in the long-short column. } \\
& \underline{t}_{i}^{x} \leq \underline{t}_{i}^{S} \text { occurred at least } j \text { times among } i=1, \ldots, 11 \text {, in the short-leg column. } \\
& |t|_{i}^{x} \leq|t|_{i}^{S} \text { occurred at least } j \text { times among } i=1, \ldots, 11 \text {, in the long-leg column. }
\end{aligned}
$$

The "combination" row reflects the frequencies with which a simulated predictor produces t-statistics satisfying the above inequalities when $R_{i, t}$ is an equally weighted combination of the 11 anomaly strategies. The final row reflects the frequencies with which the above inequalities are satisfied for 11 anomalies as well as the combination strategy. The last two columns reflect the frequencies with which the inequalities are satisfied jointly across the previous columns.

\begin{tabular}{lccccc}
\hline & $(1)$ & $(2)$ & $(3)$ & & \\
Comparisons & Long-Short & Short Leg & Long Leg & $(1)$ and $(2)$ & $(1),(2)$, and $(3)$ \\
\hline 1 anomaly & 22 & 39 & 1.2 & - & - \\
2 anomalies & 57 & 77 & 1.5 & - & - \\
3 anomalies & 124 & 146 & 1.9 & - & - \\
4 anomalies & 251 & 288 & 2.6 & - & - \\
5 anomalies & 469 & 616 & 3.7 & - & - \\
6 anomalies & 833 & 1,310 & 5.4 & - & - \\
7 anomalies & 1,460 & 2,950 & 8.5 & - & - \\
8 anomalies & 2,570 & 5,700 & 14 & - & - \\
9 anomalies & 4,740 & 11,400 & 25 & - & - \\
10 anomalies & 10,000 & 28,400 & 51 & - & 6,580 \\
11 anomalies & 28,500 & 105,000 & 143 & - & $>200,000,000^{\mathrm{a}}$ \\
Combination & 67 & 169 & 13 & 221 & - \\
11 plus the combination & 28,500 & 105,000 & 919 & 468,000 & - \\
\hline
\end{tabular}

aThere were zero cases obtained among the 200,000,000 predictors randomly generated. 


\section{Table 2}

\section{Number of Randomly Generated Predictors Required to Obtain One Predictor That Enters with the Correct Sign}

The table reports the reciprocal of the frequency with which a randomly generated predictor $x_{t}$ produces an estimate of $b$ with the predicted sign when $x_{t}$ replaces $S_{t}$ in the regression,

$$
R_{i, t}=a+b S_{t-1}+c M K T_{t}+d S M B_{t}+e H M L_{t}+u_{t},
$$

where $R_{i, t}$ is the excess return in month $t$ on an anomaly's long leg, short leg, or the difference, $S_{t}$ is the level of the investor-sentiment index of Baker and Wurgler (2006), and $M K T_{t}, S M B_{t}$ and $H M L_{t}$ are the three stock-market factors defined in Fama and French (1993). The predictor $x_{t}$ is generated as a first-order autoregression with autocorrelation equal to 0.988 , the bias-corrected estimate of the autocorrelation of $S_{t}$.

The row for $j$ anomalies reflects the frequency with which a simulated predictor produces an estimate of $b$ for at least $j$ anomalies with the predicted sign (positive in the long-short column and negative in the short-leg column). The "combination" row reflects the frequencies with which a simulated predictors produces an estimate of $b$ with the predicted sign when $R_{i, t}$ is an equally weighted combination of the 11 anomaly strategies. The last column reflects the frequencies with which the predicted signs are obtained jointly across the previous columns.

\begin{tabular}{lccc}
\hline & $\begin{array}{c}(1) \\
\text { Lomp-Short }\end{array}$ & $\begin{array}{c}(2) \\
\text { Short Leg }\end{array}$ & $(1)$ and $(2)$ \\
\hline 1 anomaly & 1.0 & 1.1 & - \\
2 anomalies & 1.1 & 1.1 & - \\
3 anomalies & 1.3 & 1.3 & - \\
4 anomalies & 1.4 & 1.4 & - \\
5 anomalies & 1.7 & 1.7 & - \\
6 anomalies & 2.0 & 2.0 & - \\
7 anomalies & 2.5 & 2.5 & - \\
8 anomalies & 3.3 & 3.3 & - \\
9 anomalies & 4.9 & 4.9 & - \\
10 anomalies & 8.8 & 8.5 & - \\
11 anomalies & 25 & 21 & - \\
Combination & 2.0 & 2.0 & 2.2 \\
11 plus the combination & 25 & 21 & 43 \\
\hline
\end{tabular}




\section{References}

Antoniou, Constantinos, John A. Doukas, and Avanidhar Subrahmanyam, 2010, Sentiment and momentum, working paper, UCLA.

Baker, M, Wurgler, J., 2006. Investor sentiment and the cross-section of stock returns. Journal of Finance 61, 1645-1680.

Baker, M., Wurgler, J., 2007. Investor sentiment in the stock market. Journal of Economic Perspectives $21,129-152$.

Baker, Malcolm, Jeffrey Wurgler, and Yu Yuan, 2012, Global, Local, and Contagious Investor Sentiment, Journal of Financial Economics 104, 272-287.

Brown, Gregory W., and Michael T. Cliff, 2004, Investor sentiment and the near-term stock market, Journal of Empirical Finance 11, 1-27.

Brown, Gregory W., and Michael T. Cliff, 2005, Investor sentiment and asset valuation, Journal of Business 78, 405-440.

Campbell, J. Y., Hilscher, J., Szilagyi, J., 2008. In search of distress risk. Journal of Finance 63, 2899-2939.

Cooper, M. J., Gulen, H., Schill, M. J., 2008. Asset growth and the cross-section of stock returns. Journal of Finance 63, 1609-1652.

Daniel, K. D., Titman, S., 2006. Market reactions to tangible and intangible information. Journal of Finance 61, 1605-1643.

Fama, E., French, K., 1993. Common risk factors in the returns on stocks and bonds. Journal of Financial Economics 33, 3-56.

Fama, E., French, K., 2006. Profitability, investment, and average returns. Journal of Financial Economics 82, 491-518.

Ferson, W., Sarkissian, S., Simin, T.T., 2003. Spurious regressions in financial economics? Journal of Finance 58, 1393-1413.

Gao, Xiaohui, Jianfeng Yu and Yu Yuan, 2010, Investor sentiment and the idiosyncratic risk puzzle, working paper, Hong Kong University.

Granger, C. W.J., Newbold,P., 1974. Spurious regressions in economics. Journal of Econometrics $4,111-120$.

Hirshleifer, D., Hou, K., Teoh, S. H., Zhang, Y., 2004. Do investors overvalue firms with bloated balance sheets. Journal of Accounting and Economics 38, 297-331.

Jegadeesh, N., Titman, S., 1993. Returns to buying winners and selling losers: implications for market efficiency. Journal of Finance 48, 65-91.

Kendall, M.G., 1954. Note on bias in the estimation of autocorrelation. Biometrika 41, 403-404. 
Keynes, J. M., 1936. The General Theory of Employment, Interest, and Money. Macmillan, London.

Lemmon M., Portniaquina, E., 2006. Consumer confidence and asset prices: some empirical evidence. Review of Financial Studies 19, 1499- 1529.

Livnat, Joshua and Christine Petrovits, 2009, Investor sentiment, post-earnings announcement drift, and accruals, working paper, New York University.

Loughran, T., Ritter, J. R., 1995. The new issues puzzle. Journal of Finance 50, 23-51.

Marriott, F.H.C., Pope, J.A., 1954. Bias in the estimation of autocorrelations. Biometrika 41, 390-402.

Miller, E. M., 1977. Risk, uncertainty and divergence of opinion. Journal of Finance 32, 1151-1168.

Novy-Marx, R., 2010. The other side of value: good growth and the gross profitability premium. Unpublished working paper. University of Chicago.

Novy-Marx, R., 2012. Pseudo-predictability in conditional asset pricing tests: Explaining anomaly performance with politics, the weather, global warming, sunspots, and the stars. Unpublished working paper. University of Rochester.

Ohlson, J. A., 1980. Financial ratios and the probabilistic prediction of bankruptcy. Journal of Accounting Research 18, 109-131.

Ritter, J. R., 1991. The long-run performance of initial public offerings. Journal of Finance 46, $3-27$.

Sloan, R.G., 1996. Do stock prices fully reflect information in accruals and cash flows about future earnings? Accounting Review 71, 289-315.

Stambaugh, R.F., 1999. Predictive regressions. Journal of Financial Economics 54, 375-421.

Stambaugh, R.F., Yu, J., and Yuan, Y., 2012. The short of it: Investor sentiment and anomalies. Journal of Financial Economics 104, 288-302.

Titman, S., Wei, K., Xie, F., 2004. Capital investments and stock returns. Journal of Financial and Quantitative Analysis 39, 677-700.

Wang, H., Yu, J., 2010. Dissecting the profitability premium. Unpublished working paper. University of Minnesota.

Xing, Y., 2008. Interpreting the value effect through the Q-theory: an empirical investigation. Review of Financial Studies 21, 1767-1795.

Yu, Jianfeng, 2009, A sentiment-based explanation of the forward premium puzzle. working paper, University of Minnesota.

Yule, G. U., 1926. Why do we sometimes get nonsense correlations between time series? A study in sampling and the nature of time series. Journal of the Royal Statistical Society 89, 1-64. 